\title{
Discovery of thermophilic Bacillales using reduced-representation genotyping for identification
}

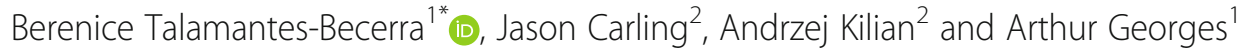

\begin{abstract}
Background: This study demonstrates the use of reduced-representation genotyping to provide preliminary identifications for thermophilic bacterial isolates. The approach combines restriction enzyme digestion and PCR with next-generation sequencing to provide thousands of short-read sequences from across the bacterial genomes. Isolates were obtained from compost, hot water systems, and artesian bores of the Great Artesian Basin. Genomic DNA was double-digested with two combinations of restriction enzymes followed by PCR amplification, using a commercial provider of DArTseq ${ }^{\text {TM }}$, Diversity Arrays Technology Pty Ltd. (Canberra, Australia). The resulting fragments which formed a reduced-representation of approximately $2.3 \%$ of the genome were sequenced. The sequence tags obtained were aligned against all available RefSeq bacterial genome assemblies by BLASTn to identify the nearest reference genome.

Results: Based on the preliminary identifications, a total of 99 bacterial isolates were identified to species level, from which 8 isolates were selected for whole-genome sequencing to assess the identification results. Novel species and strains were discovered within this set of isolates. The preliminary identifications obtained by reduced-representation genotyping, as well as identifications obtained by BLASTn alignment of the $16 \mathrm{~S}$ rRNA gene sequence, were compared with those derived from the whole-genome sequence data, using the same RefSeq sequence database for the three methods. Identifications obtained with reduced-representation sequencing agreed with the identifications provided by whole-genome sequencing in 100\% of cases. The identifications produced by BLASTn alignment of 16S rRNA gene sequence to the same database differed from those provided by whole-genome sequencing in $37.5 \%$ of cases, and produced ambiguous identifications in $50 \%$ of cases.
\end{abstract}

Conclusions: Previously, this method has been successfully demonstrated for use in bacterial identification for medical microbiology. This study demonstrates the first successful use of DArTseq ${ }^{\text {TM }}$ for preliminary identification of thermophilic bacterial isolates, providing results in complete agreement with those obtained from whole-genome sequencing of the same isolates. The growing database of bacterial genome sequences provides an excellent resource for alignment of reduced-representation sequence data for identification purposes, and as the available sequenced genomes continue to grow, the technique will become more effective.

Keywords: Bacterial identification, DArTseq, Genotyping-by-sequencing, Great Artesian Basin, Reduced-representation sequencing, Thermophiles

\footnotetext{
* Correspondence: Berenice.TalamantesBecerra@canberra.edu.au

${ }^{1}$ Institute of Applied Ecology, University of Canberra, Canberra, ACT 2601,

Australia

Full list of author information is available at the end of the article
}

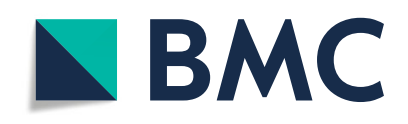

(c) The Author(s). 2020 Open Access This article is licensed under a Creative Commons Attribution 4.0 International License, which permits use, sharing, adaptation, distribution and reproduction in any medium or format, as long as you give appropriate credit to the original author(s) and the source, provide a link to the Creative Commons licence, and indicate if changes were made. The images or other third party material in this article are included in the article's Creative Commons licence, unless indicated otherwise in a credit line to the material. If material is not included in the article's Creative Commons licence and your intended use is not permitted by statutory regulation or exceeds the permitted use, you will need to obtain permission directly from the copyright holder. To view a copy of this licence, visit http://creativecommons.org/licenses/by/4.0/. The Creative Commons Public Domain Dedication waiver (http://creativecommons.org/publicdomain/zero/1.0/) applies to the data made available in this article, unless otherwise stated in a credit line to the data. 


\section{Background}

Thermophiles continue to generate interest owing to the thermostability of their enzymes, which have been adapted for use in scientific and industrial processes The proteins of thermophilic bacteria generally exhibit higher thermostability compared to those of mesophiles, in part because they tend to have stronger hydrophobic interactions amongst their amino acids than in other bacteria [1]. The ability to withstand such extreme temperatures has made the enzymes from thermophilic bacteria of particular interest for commercial, industrial and scientific applications [2-5] in areas such as pharmaceutical [6], food [7, 8] and detergent industries [9].

The classic environments in which thermophilic microorganisms occur are primarily geothermal in nature [10]. The Great Artesian Basin in South Australia has the temperature and chemical properties which are suitable for thermophiles [11-13]. Specifically, there are bores in this region with water temperatures of $90{ }^{\circ} \mathrm{C}$ or more $[14,15]$, some of them with open, running bore drains known to contain communities of thermophilic microorganisms [16]. Thermophilic bacteria are also found in other environments such as compost [17-20] and hot water systems $[21,22]$.

The isolation and discovery of thermophilic bacteria is a continuing area of research interest around the world. The identification of novel thermophilic isolates is now routinely achieved through DNA sequencing methods. Jain et al. (2018), in a high throughput analysis of 90 thousand bacterial genomes, discussed the importance of accurate estimation of genetic relatedness in species delimitation. In this context, ANI (Average Nucleotide Identity) has been considered one of the standard tools for this task. ANI is calculated as the average nucleotide identity from the set of orthologous genes identified between any two genomes. Organisms belonging to the same species are typically considered to show ANI values of $\geq 95 \%$ in pairwise comparisons [23].

Here we aim to assess reduced-representation sequencing as an alternative method for preliminary identification of isolates derived from sampling locations across Australia. A standard approach to the identification of novel bacterial strains or species utilises partial or complete 16S rRNA gene sequence as a preliminary identification method to screen for potentially novel strains or species among a set of isolates. Candidates identified from the $16 \mathrm{~S}$ rRNA gene sequencing subsequently undergo whole-genome sequencing. The use of 16S rRNA gene sequencing for bacterial identification is well established, although it has two potential limitations: firstly, in some cases it is necessary to attempt more than one set of PCR primers in order to achieve amplification from bacterial genomes of unknown taxonomic affinity, and secondly, the potential for limited resolution of identifications obtained from the $16 \mathrm{~S}$ rRNA gene sequence. For this study, we have tested a novel approach of reduced-representation sequencing for the first stage identification of bacterial isolates to identify 99 isolates from a variety of thermal sources. Additionally, we have compared the preliminary identification outcomes obtained from 16S rRNA gene sequence and reduced-representation sequencing with identifications derived from whole-genome sequence on a subset of bacterial isolates. Our method used DArTseq (Canberra, Australia) [24], one of several available methods for generating representative sequences from the genome. It uses restriction enzyme digestion followed by PCR and Illumina short-read sequencing to amplify and sequence thousands of restriction fragments as genomic representations. DArTseq ${ }^{\mathrm{Tm}}$ has been successfully used for a broad range of applications, for breeding of plants and animals [25], for assessment of genetic diversity [26-28] and for ecological genetics [29, 30]. This study represents the first usage of DArTseq ${ }^{\mathrm{Tm}}$ for identification of thermophilic bacterial isolates.

\section{Results \\ In-silico analysis of control E. coli 0157 (EDL 933) IRMM449 certified reference standard}

Reduced-representation sequence assays were performed as a control experiment on the reference standard genomic DNA of E. coli O157 (EDL 933) IRMM449 [31], with 6 technical replicates for each combination of restriction enzymes. Correct identification results were produced for all assays at the species and strain level using the Currito3.1 DNA Fragment Analysis Software [32] which was developed for this project. The mean genome coverage obtained for each method was $2.64 \%$ for PstI with HpaII and $2.34 \%$ for PstI with MseI. The mean BLASTn percentage alignment values obtained against the genome sequence of E. coli O157 (EDL 933) IRMM449, GenBank accession number CP008957.1 [31] were 99.9915 and $99.9974 \%$, respectively. The average number of restriction fragments obtained in the sequence output for each method was 2433 and 1836 fragments, respectively. Finally, the average nucleotide sequence distance (NSD) value obtained for each method was 0.000103 and 0.000040 respectively. For the PstI with HpaII enzyme combination, the average NSD values showed less than $1 \mathrm{bp}$ of difference per 10,000 bp aligned.

\section{Isolation of the strains}

A total of 99 bacterial isolates were obtained from 27 different sampling sources. Microbial growth results of 31 isolates from hot water systems and commercial composts are shown in Table 1 and microbial growth 
Table 1 Microbial growth for samples from hot water systems and commercial compost. Incubation temperature was $62.5^{\circ} \mathrm{C}$, culture media LB broth agar

\begin{tabular}{|c|c|c|c|c|}
\hline Source & Sample name & Temperature $\left(\mathrm{T}^{\circ} \mathrm{C}\right)$ & Microbial growth & No. Bacterial isolates \\
\hline \multicolumn{5}{|c|}{ Domestic hot water systems } \\
\hline & DPS1 & $60^{\circ} \mathrm{C}$ & $(+)(A),(+)(B)$ & 2 \\
\hline & DPS2 & $61.1^{\circ} \mathrm{C}$ & $(+)(A),(+)(B)$ & 2 \\
\hline & DPS3 & $62.6^{\circ} \mathrm{C}$ & $(+)$ & 1 \\
\hline & DPS4 & $62.6^{\circ} \mathrm{C}$ & $(+)(A),(+)(B)$ & 2 \\
\hline & DPS5 & $79.6^{\circ} \mathrm{C}$ & $(+)(\mathrm{A}),(+)(\mathrm{B}),(+)(\mathrm{C})$ & 3 \\
\hline & DPS6 & $57^{\circ} \mathrm{C}$ & $(+)(A),(+)(B),(+)(C)$ & 3 \\
\hline & DPS7 & $57.6^{\circ} \mathrm{C}$ & $(-)$ & \\
\hline & HTR & $84^{\circ} \mathrm{C}$ & $(++)$ & 1 \\
\hline & DHW & $60^{\circ} \mathrm{C}$ & $(+)(A),(+)(B)$ & 2 \\
\hline \multicolumn{5}{|l|}{ Commercial compost } \\
\hline & DMW & na & $(+)$ & 1 \\
\hline & MPCC & na & $(+)$ & 1 \\
\hline & NFOSA & na & $(+)(A),(+)(B),(+)(C)$ & 3 \\
\hline & MMBA & na & $(+)(A),(+)(B)$ & 2 \\
\hline & MFBB & na & $(+)(A),(+)(B),(+)(C),(+)(D)$ & 4 \\
\hline & MPCB & na & $(+)$ & 1 \\
\hline & CBSP & na & $(+)(A),(+)(B),(+)(C)$ & 3 \\
\hline Total bacterial isolates & & & & 31 \\
\hline
\end{tabular}

na = not applicable; $(-)=$ no growth was observed; $(+)=$ growth was observed; $(++)=$ strong growth was observed; (A), (B), (C) name assigned if more than one microorganism was observed

results of 68 bacterial isolates from artesian bore water and bore drains are shown in Table 2.

Sediment and water samples inoculated into culture media showed different growth depending on the source. Sediment samples collected from flowing bore drains from the Great Artesian Basin, showed heavy microbial growth at various $\mathrm{pH}$ values ranging from between 5.0 to 8.0. Microbial colonies within the inoculated agar plates sometimes showed differing morphologies, indicating the possible presence of more than one bacterial strain. Colonies with distinct morphologies were isolated individually (Table 2).

\section{Species identification}

Results obtained from the analysis of the reducedrepresentation sequences using Currito3.1 DNA Fragment Analysis Software [32] provided preliminary identification and similarity information for all isolates. An example of the report produced by Currito3.1 DNA Fragment Analysis Software [32] for the isolate MMMud_3_LB_pH8 is shown in Fig. 1. The full list of identification results of isolates obtained from hot water systems and commercial composts are shown in Table 3 and the identification results of all isolates from artesian bore water and bore drains are shown in Table 4. Colonies possessing different morphologies within a single plate were isolated and given suffix a, b, c, and d, in some cases these isolates may be duplicates. For each isolate, the nearest sequenced genome from the NCBI RefSeq database is given, along with the average BLASTn percentage identity based on the nearest genome. Percentages obtained ranged from 85.43 to $99.84 \%$. In many instances the BLASTn percentage identity against the nearest genome was $>98.00 \%$, indicating that the isolates belonged to the same species. From this set, 8 isolates were found to have a BLASTn percentage identity against the nearest genome of $<95.00 \%$, indicating potential new species [23].

A total of 16 bacterial isolates were collected from nine domestic hot water systems. Temperatures at which these were collected ranged between $60^{\circ} \mathrm{C}$ to $84^{\circ} \mathrm{C}$. A total of 15 bacterial isolates were obtained from seven commercial garden compost sources. The composting temperatures were not recorded, although the range of temperatures occurring during the high-temperature phase of the composting process has been reported as between $40{ }^{\circ} \mathrm{C}$ to $78^{\circ} \mathrm{C}$ [34]. All isolates derived from omestic hot water systems and compost were identified as belonging to the genus Geobacillus.

A total of 18 bacterial isolates were obtained from filtered water samples of artesian bores in The Great Artesian Basin. The range of temperatures at which the 
Table 2 Microbial growth for water and mud samples from the Great Artesian Basin. Incubation temperature was $62.5^{\circ} \mathrm{C}$. Culture media: LB broth agar ( $\mathrm{pH} 5, \mathrm{pH} 6.8, \mathrm{pH}$ ) and PBT pH 6.0

\begin{tabular}{|c|c|c|c|c|c|c|c|c|c|}
\hline \multirow[b]{2}{*}{ Location } & \multirow[b]{2}{*}{ Source } & \multirow[b]{2}{*}{$\begin{array}{l}\text { Temperature } \\
\left({ }^{\circ} \mathrm{C}\right)\end{array}$} & \multirow[b]{2}{*}{$\mathrm{pH}$} & \multirow[b]{2}{*}{ Sample name } & \multirow[b]{2}{*}{$\mathrm{pH} 5.0$} & \multicolumn{2}{|l|}{ LB Broth } & \multirow{2}{*}{$\begin{array}{l}\text { PBT } \\
\text { pH } 6.0\end{array}$} & \multirow{2}{*}{$\begin{array}{l}\text { No. } \\
\text { Bacterial } \\
\text { isolates }\end{array}$} \\
\hline & & & & & & $\mathrm{pH} 6.8$ & $\mathrm{pH} 8.0$ & & \\
\hline \multirow[t]{2}{*}{ Birdsville } & water & $98^{\circ} \mathrm{C}^{\mathrm{a}}$ & nd & Birdsville Bore & nd & $(+)$ & $(+)$ & $(-)$ & 2 \\
\hline & $\begin{array}{l}\text { bore } \\
\text { drain }\end{array}$ & $98^{\circ} \mathrm{C}^{\mathrm{a}}$ & nd & Birdsville mud & nd & $(+)(\mathrm{A}),(+)(\mathrm{B})$ & $(+)$ & $\begin{array}{l}(+)(\mathrm{A}),(+)(\mathrm{B}) \\
(+)(\mathrm{C})\end{array}$ & 6 \\
\hline \multirow[t]{2}{*}{ Clifton hills } & water & $80^{\circ} \mathrm{C}$ & 8.0 & CHfil & $(-)$ & $(-)$ & $(+)(A)$ & $(-)$ & 1 \\
\hline & $\begin{array}{l}\text { bore } \\
\text { drain }\end{array}$ & $80^{\circ} \mathrm{C}$ & nd & CHMUD & $(+)$ & $(++)$ & $\begin{array}{l}(++)(\mathrm{A}) \\
(++)(\mathrm{B})\end{array}$ & $(++)$ & 4 \\
\hline \multirow[t]{2}{*}{ Mount Gason } & water & $80^{\circ} \mathrm{C}$ & 8.0 & MtGfil & nd & $(+)(\mathrm{A}),(+)(\mathrm{B})$ & $(+)(A)$ & $(+)(A)$ & 4 \\
\hline & $\begin{array}{l}\text { bore } \\
\text { drain }\end{array}$ & $80^{\circ} \mathrm{C}$ & nd & $\begin{array}{l}\text { Mt.GODS / Mt. } \\
\text { GMUD }\end{array}$ & $\begin{array}{l}(+)(A), \\
(+)(B)\end{array}$ & $\begin{array}{l}(+)(\mathrm{A}),(+)(\mathrm{B}),(+)(\mathrm{C}),(+)(\mathrm{D}),(+)(\mathrm{E}), \\
(+)(\mathrm{F}),(+)(\mathrm{G})\end{array}$ & $\begin{array}{l}(+)(\mathrm{A}),(+)(\mathrm{B}) \\
(+)(\mathrm{C})\end{array}$ & $(+)$ & 13 \\
\hline \multirow[t]{9}{*}{ Mirra Mita } & water & $79^{\circ} \mathrm{C}$ & 8.0 & MMfil & nd & $(+)$ & $(-)$ & $(-)$ & 1 \\
\hline & $\begin{array}{l}\text { bore } \\
\text { drain }\end{array}$ & $79^{\circ} \mathrm{C}$ & nd & MMMUD1 & $(-)$ & $(+)$ & $(-)$ & $(+)$ & 2 \\
\hline & $\begin{array}{l}\text { bore } \\
\text { drain }\end{array}$ & $68^{\circ} \mathrm{C}$ & nd & MMMUD2 & $(++)$ & $(++)$ & $(-)$ & $(-)$ & 2 \\
\hline & $\begin{array}{l}\text { bore } \\
\text { drain }\end{array}$ & $74^{\circ} \mathrm{C}$ & nd & MMMUD3 & $(-)$ & $(++)$ & $(+)(\mathrm{A})$ & $(+)$ & 3 \\
\hline & $\begin{array}{l}\text { bore } \\
\text { drain }\end{array}$ & $62^{\circ} \mathrm{C}$ & nd & MMMUD4 & $(-)$ & $(+)(\mathrm{A}),(+)(\mathrm{B})$ & $(+)(\mathrm{A}),(+)(\mathrm{B})$ & $(+)$ & 5 \\
\hline & $\begin{array}{l}\text { bore } \\
\text { drain }\end{array}$ & $66^{\circ} \mathrm{C}$ & nd & MMMUD5 & $(-)$ & $(+)(\mathrm{A}),(+)(\mathrm{B})$ & $(-)$ & $(-)$ & 2 \\
\hline & $\begin{array}{l}\text { bore } \\
\text { drain }\end{array}$ & $50^{\circ} \mathrm{C}$ & nd & MMMUD6 & $(-)$ & $(+)(\mathrm{A}),(+)(\mathrm{B})$ & $(-)$ & $(-)$ & 2 \\
\hline & $\begin{array}{l}\text { bore } \\
\text { drain }\end{array}$ & $39^{\circ} \mathrm{C}$ & nd & MMMUD7 & $(-)$ & $(++)$ & $(-)$ & $(-)$ & 1 \\
\hline & $\begin{array}{l}\text { bore } \\
\text { drain }\end{array}$ & $74^{\circ} \mathrm{C}$ & nd & MMMUD8 & $(+)$ & $(-)$ & $(-)$ & $(-)$ & 1 \\
\hline \multirow[t]{2}{*}{$\begin{array}{l}\text { Mungerannie } \\
\text { station }\end{array}$} & water & $78^{\circ} \mathrm{C}$ & 7.2 & $\begin{array}{l}\text { Mgnhotfil / } \\
\text { MgnCCG }\end{array}$ & $(+)$ & $(+)$ & $(+)$ & $(-)$ & 3 \\
\hline & $\begin{array}{l}\text { bore } \\
\text { drain }\end{array}$ & $60^{\circ} \mathrm{C}$ & nd & MCWH & $(-)$ & $(+)(\mathrm{A}),(+)(\mathrm{B})$ & $(-)$ & $(-)$ & 2 \\
\hline Mulka & soil & $38^{\circ} \mathrm{C}$ & nd & $M R$ & $(-)$ & $(+)(A)$ & $(+)(A)$ & $(-)$ & 2 \\
\hline \multirow[t]{2}{*}{ Kopperamanna } & water & $60^{\circ} \mathrm{C}$ & 8.5 & Efil & $(-)$ & $(+)(\mathrm{A}),(+)(\mathrm{B})$ & $(-)$ & $(++)$ & 3 \\
\hline & soil & $38^{\circ} \mathrm{C}$ & nd & $\mathrm{ECO} 3$ & $(-)$ & $(-)$ & $(-)$ & $(++)$ & 1 \\
\hline $\begin{array}{l}\text { Etadunna } \\
\text { station }\end{array}$ & water & $77.9^{\circ} \mathrm{C}$ & 8.5 & Kanufil & nd & $(+)$ & $(-)$ & $(-)$ & 1 \\
\hline Dulkaninna & water & $47.8^{\circ} \mathrm{C}$ & 8.5 & Dulfil & nd & $(+)(+)$ & $(-)$ & $(-)$ & 1 \\
\hline \multirow[t]{2}{*}{ Clayton station } & water & $34^{\circ} \mathrm{C}$ & 8.5 & Clfil & nd & $(+)(A),(+)(B)$ & $(-)$ & $(-)$ & 2 \\
\hline & $\begin{array}{l}\text { bore } \\
\text { drain }\end{array}$ & $34^{\circ} \mathrm{C}$ & nd & CLB & $(-)$ & $(+)$ & $(-)$ & $(-)$ & 1 \\
\hline \multirow[t]{2}{*}{ Lake Harry } & water & $46^{\circ} \mathrm{C}$ & 8.5 & LHfil & nd & $(-)$ & $(-)$ & $(-)$ & \\
\hline & $\begin{array}{l}\text { bore } \\
\text { drain }\end{array}$ & $46^{\circ} \mathrm{C}$ & nd & LHMUD & $(-)$ & $(+)(\mathrm{A}),(+)(\mathrm{B})$ & $(-)$ & $(+)$ & 3 \\
\hline $\begin{array}{l}\text { Total bacterial } \\
\text { isolates }\end{array}$ & & & & & & & & & 68 \\
\hline
\end{tabular}

$\left({ }^{\mathrm{a}}\right)$ Based on published temperature (Habermehl and Pestov 2002 [14])

nd = not determined; $(-)=$ no growth was observed; $(+)=$ growth was observed; $(++)=$ strong growth was observed; (A), (B), (C) name assigned if more than one microorganism was observed 


\section{Identification report}

Sample name: MMMud_3_LB_pH8 Collection date: 25/11/15 Location: Mirra Mita

\begin{tabular}{|c|c|c|c|c|}
\hline $\begin{array}{l}\text { First candidate closest } \\
\text { match }\end{array}$ & $\begin{array}{c}\text { Mean } \\
\text { BLAST \% } \\
\text { identity }\end{array}$ & $\begin{array}{l}\text { NSD } \\
\text { value }\end{array}$ & $\begin{array}{c}\text { Genome } \\
\text { coverage } \\
(\%)\end{array}$ & $\begin{array}{l}\text { Total number } \\
\text { of sequence } \\
\text { tags aligned }\end{array}$ \\
\hline $\begin{array}{c}\text { Geobacillus subterraneus } \\
\text { PSS2 N671 }\end{array}$ & $98.14 \%$ & 0.01122 & $1.50 \%$ & 953 \\
\hline
\end{tabular}

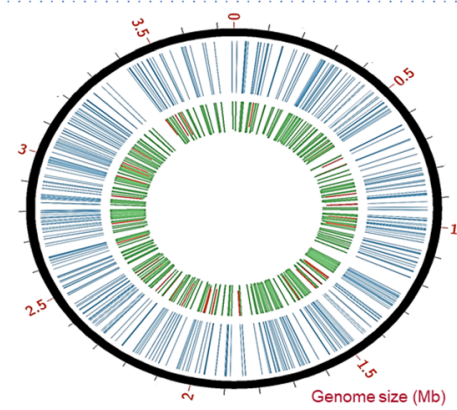

Circular plot showing the location of tags aligned to genome

Geobacillus subterraneus PSS2 N671 (NZ_JQMN01000001).

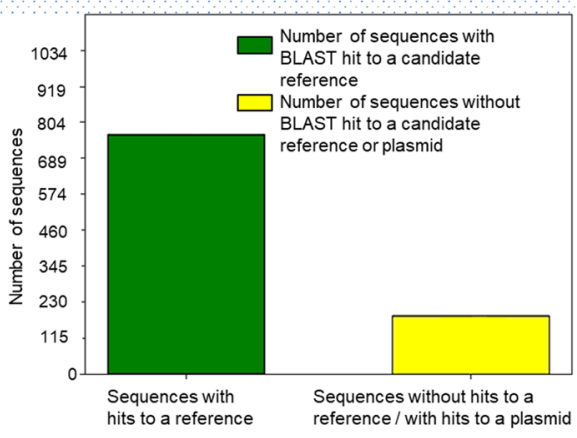

Total number of sequence tags obtained showing alignment information.

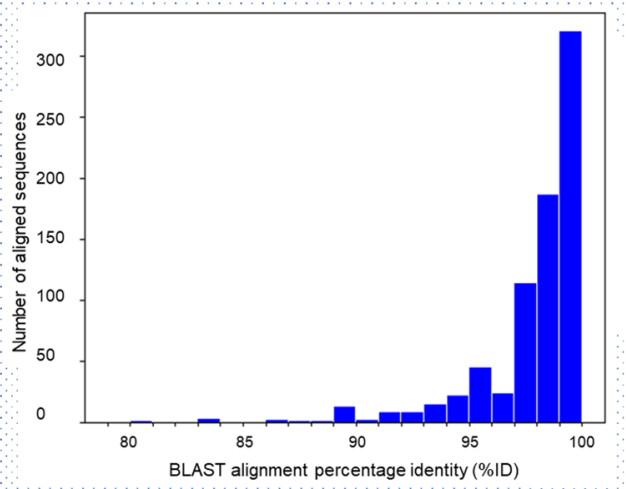

Histogram showing BLAST alignment $\%$ identity of all BLAST alignments against the candidate references.

The $X$ axis shows the BLAST alignment $\%$ identity (in 1\% bins) and the $Y$ axis shows the number of aligned sequences.

Fig. 1 Extract of report generated by bioinformatics pipeline Currito3.1 DNA Fragment Analyser [32] for sample MMMud_3_LB_ph8. This image shows the first candidate closest match to the sample. The circular graph plotted in Circos [33] shows the BLAST alignment position against the reference genome. The outer black circle represents the candidate reference genome with size indicated in megabases (Mb); the middle blue circle shows aligned sequenced fragments obtained by complexity-reduced genotyping and the Inner green / red circle shows the percentage identity of the alignments, in which values below 95\% are red and values equal or above $95 \%$ are green. The bar plots show the sequences obtained with and without BLAST alignments against the best reference, in which the $X$ axis shows the sequences classification of sequences with and without hits to a reference or plasmid and the $Y$ axis shows the total number of sequences. The histogram shows the percentage identity of BLAST alignments against the candidate reference, where the $X$ axis shows the BLAST alignment percentage identity highest to lowest and the $Y$ axis has the number of aligned sequences 
Table 3 Bacterial identification results for isolates obtained from hot water systems and commercial compost, showing nearest matches based on BLAST alignment of complexity-reduced genotyping fragments. Average BLASTn percentage identity, nucleotide sequence distance values to best matches and genome coverage percentage are shown

\begin{tabular}{|c|c|c|c|c|c|}
\hline Source & $\begin{array}{l}\text { Sample } \\
\text { name }\end{array}$ & Closest match & $\begin{array}{l}\text { Average \% } \\
\text { identity }\end{array}$ & $\begin{array}{l}\text { Nucleotide sequence distance } \\
\text { (NSD) }\end{array}$ & $\begin{array}{l}\text { Genome coverage } \\
(\%)\end{array}$ \\
\hline \multicolumn{6}{|c|}{ Domestic hot water systems } \\
\hline & DHWa & Geobacillus sp. 8 & 99.27 & 0.00446 & 2.28 \\
\hline & $\mathrm{DHWb}$ & Geobacillus sp. 8 & 98.84 & 0.00719 & 2.12 \\
\hline & DSP1a & Geobacillus lituanicus strain N-3 & 98.18 & 0.01177 & 1.80 \\
\hline & DPS1b & Geobacillus lituanicus strain N-3 & 98.11 & 0.01174 & 1.80 \\
\hline & DSP2a & Geobacillus lituanicus strain N-3 & 98.58 & 0.00838 & 3.25 \\
\hline & DSP2b & Geobacillus lituanicus strain N-3 & 98.58 & 0.00855 & 3.23 \\
\hline & DSP3 & Geobacillus lituanicus strain N-3 & 98.61 & 0.00837 & 3.18 \\
\hline & DSP4a & Geobacillus lituanicus strain N-3 & 98.55 & 0.00871 & 3.29 \\
\hline & DSP4b & Geobacillus lituanicus strain N-3 & 98.60 & 0.00849 & 3.27 \\
\hline & DSP5a & Geobacillus lituanicus strain N-3 & 98.29 & 0.01041 & 4.32 \\
\hline & DSP5b & Geobacillus lituanicus strain N-3 & 98.33 & 0.01023 & 4.29 \\
\hline & DPS5c & Geobacillus lituanicus strain N-3 & 98.35 & 0.01006 & 4.27 \\
\hline & DSP6a & Geobacillus lituanicus strain N-3 & 98.51 & 0.00876 & 3.29 \\
\hline & DSP6b & Geobacillus sp. MAS1 T260 & 98.37 & 0.00982 & 2.06 \\
\hline & DSP6C & Geobacillus sp. MAS1 T260 & 98.35 & 0.00993 & 2.08 \\
\hline & HTR & Geobacillus sp. MAS1 T260 & 98.29 & 0.01025 & 2.02 \\
\hline \multicolumn{6}{|c|}{ Commercial compost } \\
\hline & DMW1 & Geobacillus thermoleovorans strain ID-1 & 99.66 & 0.00189 & 4.54 \\
\hline & CBSPa & $\begin{array}{l}\text { Geobacillus thermodenitrificans strain } \\
\text { G11MC16 }\end{array}$ & 99.39 & 0.00345 & 2.03 \\
\hline & $\mathrm{CBSPb}$ & $\begin{array}{l}\text { Geobacillus thermodenitrificans strain } \\
\text { G11MC16 }\end{array}$ & 99.84 & 0.00099 & 2.10 \\
\hline & CBSPC & $\begin{array}{l}\text { Geobacillus thermodenitrificans strain } \\
\text { G11MC16 }\end{array}$ & 99.83 & 0.00105 & 2.19 \\
\hline & MFBBa & Geobacillus galactosidasius strain DSM 18751 & 98.97 & 0.00648 & 1.14 \\
\hline & MFBBb & Geobacillus thermodenitrificans strain T12 & 99.59 & 0.00248 & 1.63 \\
\hline & MFBBC & Geobacillus thermodenitrificans strain JSC_T9a & 99.66 & 0.00212 & 1.10 \\
\hline & MFBBd & Geobacillus galactosidasius strain DSM 18751 & 98.65 & 0.00804 & 1.14 \\
\hline & MMBAa & Geobacillus galactosidasius strain DSM 18751 & 99.00 & 0.00671 & 1.28 \\
\hline & MMBAb & Geobacillus galactosidasius strain DSM 18751 & 98.92 & 0.00674 & 1.12 \\
\hline & MPCB & Geobacillus thermodenitrificans strain T12 & 99.58 & 0.00254 & 1.59 \\
\hline & MPCC & $\begin{array}{l}\text { Geobacillus thermodenitrificans strain } \\
\text { G11MC16 }\end{array}$ & 99.56 & 0.00267 & 1.73 \\
\hline & NFOSA1 & $\begin{array}{l}\text { Geobacillus thermodenitrificans strain } \\
\text { G11MC16 }\end{array}$ & 99.83 & 0.00106 & 2.11 \\
\hline & NFOSA2 & Geobacillus sp. 8 & 98.69 & 0.00809 & 1.85 \\
\hline & NFOSA3 & Geobacillus galactosidasius strain DSM 18751 & 98.85 & 0.00725 & 1.14 \\
\hline
\end{tabular}

water samples were collected was between $34{ }^{\circ} \mathrm{C}$ to $98{ }^{\circ} \mathrm{C}$. The $\mathrm{pH}$ values ranged from 7.2 to 8.5 . From the total, 15 bacterial isolates belonged to the genus Anoxybacillus and three isolates belonged to the genus Geobacillus.
A total of 50 bacterial isolates were obtained from artesian bore drains in the Great Artesian Basin. Artesian bore drain sediments produced the greatest diversity of strains in this study. The temperatures at which sediment was collected ranged between $34{ }^{\circ} \mathrm{C}$ to $98^{\circ} \mathrm{C}$. A 
Table 4 Bacterial identification results for isolates from the Great Artesian Basin, showing nearest matches based on BLAST alignment of complexity-reduced genotyping fragments. Average BLAST percentage identity, nucleotide sequence distance values to best matches and genome coverage percentage are shown

\begin{tabular}{|c|c|c|c|c|c|}
\hline Location & Sample name & Closest match & $\begin{array}{l}\text { Average \% } \\
\text { identity }\end{array}$ & $\begin{array}{l}\text { Nucleotide sequence } \\
\text { distance (NSD) }\end{array}$ & $\begin{array}{l}\text { Genome coverage } \\
(\%)\end{array}$ \\
\hline \multicolumn{6}{|l|}{ Birdsville } \\
\hline & B_fil_LB_pH6.8_a & Anoxybacillus ayderensis strain $\mathrm{AB} 04$ & 98.42 & 0.00937 & 2.15 \\
\hline & B_fil_LB_pH6.8_b & Anoxybacillus ayderensis strain $\mathrm{AB} 04$ & 98.33 & 0.00985 & 2.16 \\
\hline & B_mud_LB_pH6.8_a & $\begin{array}{l}\text { Anoxybacillus suryakundensis strain DSM } \\
27374\end{array}$ & 94.92 & 0.02571 & 1.43 \\
\hline & B_mud_LB_pH6.8_b & $\begin{array}{l}\text { Anoxybacillus suryakundensis strain DSM } \\
27374\end{array}$ & 94.96 & 0.02496 & 1.44 \\
\hline & B_mud_LB_pH8 & Geobacillus vulcani PSS1 N685 & 99.40 & 0.00389 & 2.08 \\
\hline & B_mud_PBT_pH6.0_a & Anoxybacillus gonensis strain G2 & 96.97 & 0.01787 & 1.43 \\
\hline & B_mud_PBT_pH6.0_b & Geobacillus sp. 8 & 98.60 & 0.00858 & 1.79 \\
\hline & B_mud_PBT_pH6.0_c & Anoxybacillus kamchatkensis strain G10 & 97.78 & 0.01344 & 3.01 \\
\hline \multicolumn{6}{|c|}{ Clifton hills } \\
\hline & CHfil_LB_pH8 & Anoxybacillus flavithermus AK1 & 99.08 & 0.00577 & 3.32 \\
\hline & CHMud_LB_pH5 & Anoxybacillus sp. 103 & 98.33 & 0.00964 & 1.26 \\
\hline & CHMud_LB_pH6.8 & Geobacillus sp. 46C-Ila & 98.53 & 0.01127 & 2.16 \\
\hline & CHMud_LB_pH8 & Anoxybacillus sp. 103 & 98.43 & 0.00903 & 1.33 \\
\hline & CHMud_PBT_pH6.0 & Anoxybacillus sp. BCO1 LR68 & 98.19 & 0.01060 & 1.62 \\
\hline \multicolumn{6}{|c|}{ Mount Gason } \\
\hline & MtGfil_LB_pH6.8_a & Anoxybacillus ayderensis strain $\mathrm{AB} 04$ & 98.42 & 0.00951 & 2.32 \\
\hline & MtGfil_LB_pH6.8_b & Anoxybacillus sp. BCO1 LR68 & 95.49 & 0.02693 & 1.88 \\
\hline & MtGfil_LB_pH8 & Anoxybacillus ayderensis strain $\mathrm{AB} 04$ & 98.21 & 0.01065 & 2.36 \\
\hline & MtGfil_PBT_pH6.0 & Anoxybacillus kamchatkensis strain G10 & 94.89 & 0.02937 & 2.19 \\
\hline & Mt_GMud_LB_pH5 & Anoxybacillus flavithermus AK1 & 97.39 & 0.01570 & 3.51 \\
\hline & Mt_GMud_LB_pH6.8_a & Anoxybacillus sp. BCO1 LR68 & 97.89 & 0.01101 & 2.02 \\
\hline & Mt_GMud_LB_pH6.8_b & Anoxybacillus kamchatkensis strain G10 & 99.34 & 0.00434 & 3.45 \\
\hline & Mt_GMud_LB_pH6.8_c & Anoxybacillus flavithermus AK1 & 99.06 & 0.00561 & 3.37 \\
\hline & Mt_GMud_LB_pH8 & Anoxybacillus kamchatkensis strain G10 & 99.37 & 0.00424 & 3.44 \\
\hline & Mt_GMud_PBT_pH6.0 & Anoxybacillus ayderensis strain $\mathrm{AB} 04$ & 96.60 & 0.02055 & 2.48 \\
\hline & Mt_GODS_LB_pH5 & Geobacillus thermoleovorans strain ID-1 & 99.01 & 0.00600 & 3.60 \\
\hline & Mt_GODS_LB_pH6.8_a & Anoxybacillus ayderensis strain $\mathrm{AB} 04$ & 98.41 & 0.00921 & 2.18 \\
\hline & $\begin{array}{l}\text { Mt_GODSa_LB_pH6.8_ } \\
\text { b }\end{array}$ & Geobacillus thermoleovorans strain ID-1 & 97.18 & 0.01851 & 2.97 \\
\hline & $\underset{c}{M t}$ MtGODSb_LB_pH6.8_ & Anoxybacillus ayderensis strain AB04 & 98.36 & 0.00918 & 2.31 \\
\hline & $\begin{array}{l}\text { Mt_GODSC_LB_pH6.8_ } \\
\text { d }\end{array}$ & Anoxybacillus ayderensis strain $\mathrm{AB} 04$ & 98.31 & 0.00937 & 2.27 \\
\hline & Mt_GODSc_LB_pH8_a & Anoxybacillus kamchatkensis strain G10 & 96.52 & 0.02288 & 3.22 \\
\hline & Mt_GODSa_LB_pH8_b & Anoxybacillus ayderensis strain $\mathrm{AB} 04$ & 98.25 & 0.00969 & 2.33 \\
\hline \multicolumn{6}{|c|}{ Mirra Mita } \\
\hline & MMfil_LB_25/11/15 & Anoxybacillus flavithermus AK1 & 98.10 & 0.01448 & 3.84 \\
\hline & MMMud_1_LB_pH6.8 & Geobacillus subterraneus PSS2 N671 & 98.15 & 0.01108 & 1.52 \\
\hline & MMMud_1_PBT_pH6.0 & Geobacillus sp. MAS1 T260 & 97.74 & 0.01651 & 1.12 \\
\hline & MMMud_2_LB_pH5 & Geobacillus subterraneus PSS2 N671 & 96.10 & 0.02497 & 1.74 \\
\hline & MMMud_2_LB_pH6.8 & Anoxybacillus kamchatkensis strain G10 & 94.41 & 0.03313 & 2.31 \\
\hline
\end{tabular}


Table 4 Bacterial identification results for isolates from the Great Artesian Basin, showing nearest matches based on BLAST alignment of complexity-reduced genotyping fragments. Average BLAST percentage identity, nucleotide sequence distance values to best matches and genome coverage percentage are shown (Continued)

\begin{tabular}{|c|c|c|c|c|c|}
\hline Location & Sample name & Closest match & $\begin{array}{l}\text { Average \% } \\
\text { identity }\end{array}$ & $\begin{array}{l}\text { Nucleotide sequence } \\
\text { distance (NSD) }\end{array}$ & $\begin{array}{l}\text { Genome coverage } \\
(\%)\end{array}$ \\
\hline & MMMud_3_LB_pH6.8 & Geobacillus subterraneus PSS2 N671 & 98.06 & 0.01171 & 1.55 \\
\hline & MMMud_3_LB_pH8 & Geobacillus subterraneus PSS2 N671 & 98.14 & 0.01122 & 1.49 \\
\hline & MMMud_3_PBT_pH6.0 & Geobacillus jurassicus NBRC 107829 & 99.05 & 0.00721 & 1.49 \\
\hline & MMMud_4_LB_pH6.8_a & Anoxybacillus gonensis strain G2 & 99.26 & 0.00500 & 2.81 \\
\hline & MMMud_4_LB_pH6.8_b & Anoxybacillus gonensis strain G2 AG-1 & 99.65 & 0.00358 & 1.87 \\
\hline & MMMud_4_LB_pH8_a & Anoxybacillus gonensis strain G2 AG-1 & 99.49 & 0.00417 & 1.87 \\
\hline & MMMud_4_LB_pH8_b & Anoxybacillus ayderensis strain AB04 & 98.41 & 0.00908 & 2.25 \\
\hline & MMMud_4_PBT_pH6.0 & Geobacillus subterraneus PSS2 N671 & 98.10 & 0.01148 & 1.38 \\
\hline & MMMud_5_LB_pH6.8_a & Geobacillus thermoleovorans strain ID-1 & 99.60 & 0.00221 & 3.60 \\
\hline & MMMud_5_LB_pH6.8_b & Geobacillus sp. 8 & 99.29 & 0.00443 & 2.39 \\
\hline & MMMud_6_LB_pH6.8_a & Geobacillus thermoleovorans strain ID-1 & 99.25 & 0.00438 & 3.58 \\
\hline & MMMud_6_LB_pH6.8_b & Geobacillus kaustophilus strain Et7/4 LG52 & 85.43 & 0.09046 & 8.38 \\
\hline & MMMud_7_LB_pH5 & Geobacillus thermoleovorans strain ID-1 & 95.90 & 0.02818 & 2.53 \\
\hline & MMMud_8_LB_pH5 & Geobacillus vulcani PSS1 N685 & 97.59 & 0.01704 & 2.12 \\
\hline \multicolumn{6}{|c|}{ Mungerannie station } \\
\hline & MgnHotfil_LB_pH6.8 & Geobacillus vulcani PSS1 N685 & 99.40 & 0.00403 & 1.88 \\
\hline & Mgn_CCG_LB_pH5 & Anoxybacillus gonensis strain G2 AG-1 & 95.60 & 0.02500 & 1.68 \\
\hline & Mgn_CCG_LB_pH8 & Anoxybacillus flavithermus AK1 & 98.93 & 0.00522 & 3.54 \\
\hline & MCWH_LB_pH6.8 & Anoxybacillus flavithermus AK1 & 99.04 & 0.00473 & 3.52 \\
\hline & MCWH_LB_pH8 & Brevibacillus thermoruber PM1 N690 & 94.13 & 0.06061 & 2.92 \\
\hline \multicolumn{6}{|l|}{ Mulka } \\
\hline & MR_LB_pH6.8 & Geobacillus kaustophilus GBlys & 99.86 & 0.00100 & 1.73 \\
\hline & MR_LB_pH8 & Geobacillus thermodenitrificans strain OS27 & 99.64 & 0.00212 & 1.41 \\
\hline \multicolumn{6}{|c|}{ Kopperamanna } \\
\hline & Efil_LB_pH6.8_a & Anoxybacillus ayderensis strain AB04 & 98.40 & 0.00959 & 2.30 \\
\hline & Efil_LB_pH6.8_b & Geobacillus thermoleovorans strain ID-1 & 95.82 & 0.02610 & 2.71 \\
\hline & Efil_PBT_pH6.0 & Anoxybacillus kamchatkensis strain G10 & 94.90 & 0.02852 & 2.23 \\
\hline & ECO3_PBT_pH6.0 & Geobacillus thermoleovorans strain KCTC 3570 & 98.08 & 0.01121 & 2.05 \\
\hline \multicolumn{6}{|c|}{ Etadunna station } \\
\hline & Kanufil_LB_pH6.8 & Geobacillus vulcani PSS1 N685 & 99.39 & 0.00394 & 1.94 \\
\hline \multicolumn{6}{|c|}{ Dulkaninna } \\
\hline & Dufil_LB_pH6.8 & Anoxybacillus kamchatkensis strain G10 & 94.92 & 0.02867 & 2.23 \\
\hline \multicolumn{6}{|c|}{ Clayton station } \\
\hline & Clfil_LB_pH6.8 & Anoxybacillus ayderensis strain AB04 & 98.43 & 0.00933 & 2.30 \\
\hline & Clfil_LB_pH6.8 & Anoxybacillus ayderensis strain $\mathrm{AB} 04$ & 94.36 & 0.03240 & 2.29 \\
\hline & CLB_LB_pH6.8 & Anoxybacillus sp. BCO1 LR68 & 97.34 & 0.01678 & 1.30 \\
\hline \multicolumn{6}{|c|}{ Lake Harry } \\
\hline & LH_Mud_LB_pH6.8_a & Anoxybacillus gonensis strain G2 AG-1 & 99.57 & 0.00379 & 1.88 \\
\hline & LH_Mud_LB_pH6.8_b & Geobacillus jurassicus NBRC 107829 & 98.34 & 0.01092 & 1.73 \\
\hline & LH_Mud_PBT_pH6.0 & Geobacillus thermoleovorans strain ID-1 & 99.04 & 0.00608 & 3.57 \\
\hline
\end{tabular}


total of 26 bore drain bacterial isolates belonged the genus Anoxybacillus, 23 isolates belonged to the genus Geobacillus and one isolate belonged to the genus Brevibacillus.

\section{Whole-genome sequencing}

To test the accuracy of preliminary identifications produced by reduced-representation sequencing, eight samples were selected for whole-genome sequencing. The eight genome assemblies were chosen to form three groups, A, B and C, based on the similarity to the nearest sequenced reference, as determined by the reducedrepresentation sequence analysis. Group A included isolates for which the identifications showed high similarity (99.4-99.64\%) to existing sequenced genomes. Group B included isolates with moderate relatedness (98.1498.85\%) to their nearest identified genome assemblies, and group C contained isolates more distantly related $(<$ 94.13\%) to any of the sequenced genome assemblies, representing potential new species. The assembly statistics for the draft genomes of the eight bacterial isolates are shown in Table 5 . The identification results obtained from progressiveMauve $[35,36]$ alignment of the eight draft genomes were compared with those derived from the reduced-representation sequence tags, and with the identifications based on 16S rRNA gene sequence alignments. These results are shown in Table 6. The results presented in this table show that identifications obtained with reduced-representation sequencing agreed with the identifications provided by whole-genome sequencing in $100 \%$ of cases. The identifications produced by BLASTn alignment of $16 \mathrm{~S}$ rRNA gene sequence to the same database differed from those provided by whole-genome sequencing in $37.5 \%$ of cases, and produced ambiguous identifications in $50 \%$ of cases.

\section{Discussion}

The results showed a complete agreement of the progressiveMauve [35, 36] whole-genome identifications and those obtained from the reduced-representation sequence alignments for all eight bacterial isolates. ProgressiveMauve $[35,36]$ can perform comparative genome alignment of two or more genomes, identifying and aligning conserved genomic DNA regions. ProgressiveMauve $[35,36]$ identifies locally colinear blocks (LCBs), which are blocks of unbroken sequence homology between genomes. The progressiveMauve [35, 36] algorithm uses an iterative process to identify and refine the boundaries of LCBs identified between genomes. ProgressiveMauve $[35,36]$ alignment is able to recognize homologous regions in the presence of multiple complex rearrangements and provides a valuable tool for analysis of sequence homology between species and strains [35, 36]. Similarity profile values, corresponding to the average level of nucleotide sequence conservation within regions of local alignment, are calculated by progressiveMauve $[35,36]$ to be inversely proportional to the average alignment column entropy within the region [35]. To determine a pairwise similarity value between genomes, each of the eight genome assemblies was aligned against the complete set of available RefSeq Anoxybacillus Geobacillus and Brevibacillus assemblies in a pairwise manner. The mean similarity profile value from each progressiveMauve $[35,36]$ pairwise alignment was calculated. These values provide a comparative measure of the similarity of each pair of genomes, averaged over all aligned sequence regions between the genome pair.

Table 5 Whole genome sequencing assembly statistics and nearest relative based on progressiveMAUVE [35, 36]

\begin{tabular}{|c|c|c|c|c|c|c|c|c|c|}
\hline Group & Sample name & $\begin{array}{l}\text { GenBank } \\
\text { accession }\end{array}$ & Reads & contigs & $\begin{array}{l}\text { Largest } \\
\text { contig }\end{array}$ & $\begin{array}{l}\text { Total } \\
\text { length }\end{array}$ & N50 & $\begin{array}{l}\mathrm{GC} \\
(\%)\end{array}$ & $\begin{array}{l}\text { Nearest relative based } \\
\text { on MAUVE }\end{array}$ \\
\hline \multirow[t]{2}{*}{$\bar{A}$} & MR_LB_pH8 & SDLB00000000 & $\begin{array}{l}3,295 \\
260\end{array}$ & 153 & $\begin{array}{l}166 \\
175\end{array}$ & $\begin{array}{l}3,592 \\
399\end{array}$ & 61,995 & 48.89 & $\begin{array}{l}\text { Geobacillus thermodenitrificans strain } \\
\text { KCTC3902 }\end{array}$ \\
\hline & B_mud_LB_pH8 & SDLA00000000 & 275,247 & 60 & $\begin{array}{l}765 \\
875\end{array}$ & $\begin{array}{l}3,434 \\
851\end{array}$ & $\begin{array}{l}188 \\
577\end{array}$ & 52.02 & Geobacillus vulcani PSS1 \\
\hline \multirow[t]{5}{*}{ B } & NFOSA3 & SDLE00000000 & $\begin{array}{l}2,067 \\
951\end{array}$ & 111 & $\begin{array}{l}273 \\
724\end{array}$ & $\begin{array}{l}3,334 \\
687\end{array}$ & 72,143 & 42.13 & Geobacillus galactosidasius strain DSM 18751 \\
\hline & DSP4a & SDLD00000000 & 885,071 & 188 & $\begin{array}{l}271 \\
123\end{array}$ & $\begin{array}{l}3,273 \\
238\end{array}$ & 58,160 & 52.32 & Geobacillus lituanicus strain N-3 \\
\hline & CHMud_LB_pH8 & SDLG00000000 & 636,456 & 67 & $\begin{array}{l}226 \\
012\end{array}$ & $\begin{array}{l}2,712 \\
590\end{array}$ & $\begin{array}{l}137 \\
053\end{array}$ & 41.81 & Anoxybacillus sp. 103 \\
\hline & Efil_LB_pH6.8 & SDLH00000000 & 777,211 & 53 & $\begin{array}{l}596 \\
376\end{array}$ & $\begin{array}{l}2,794 \\
302\end{array}$ & $\begin{array}{l}321 \\
229\end{array}$ & 41.90 & Anoxybacillus ayderensis strain AB04 \\
\hline & $\begin{array}{l}\text { MMMud_3_LB_ } \\
\text { pH8 }\end{array}$ & SDLC00000000 & $\begin{array}{l}1,174 \\
246\end{array}$ & 1196 & $\begin{array}{l}233 \\
455\end{array}$ & $\begin{array}{l}4,372 \\
943\end{array}$ & 60,623 & 55.27 & Geobacillus subterraneus PSS2 \\
\hline $\mathrm{C}$ & MCWH_LB_pH8 & SDLF00000000 & $\begin{array}{l}1,349 \\
927\end{array}$ & 124 & $\begin{array}{l}345 \\
578\end{array}$ & $\begin{array}{l}3,934 \\
072\end{array}$ & $\begin{array}{l}177 \\
957\end{array}$ & 56.20 & Anoxybacillus flavithermus strain B4168 \\
\hline
\end{tabular}


Table 6 Comparison of bacterial identification methods showing percentage identity for complexity-reduced genotyping based on BLASTn alignment; whole-genome sequencing best matches using progressiveMauve [35, 36] alignment tool; and the best matches obtained with $16 \mathrm{~S}$ rRNA gene alignment, including multiple results per sample with equal highest bitscore and percentage identity

\begin{tabular}{|c|c|c|c|c|c|c|c|}
\hline Group & $\begin{array}{l}\text { Sample } \\
\text { name }\end{array}$ & $\begin{array}{l}\text { Complexity-reduced } \\
\text { genotyping best match }\end{array}$ & $\% \mathrm{ID}$ & $\begin{array}{l}\text { Whole genome sequencing } \\
\text { best match result }\end{array}$ & $\begin{array}{l}\text { Mauve mean } \\
\text { similarity profile value }\end{array}$ & $\begin{array}{l}16 \mathrm{~s} \text { rRNA in silico best match } \\
\text { result }\end{array}$ & $\% \mathrm{ID}$ \\
\hline \multirow[t]{5}{*}{ A } & MR_LB_pH8 & $\begin{array}{l}\text { Geobacillus } \\
\text { thermodenitrificans strain } \\
\text { OS } 27\end{array}$ & 99.64 & $\begin{array}{l}\text { Geobacillus } \\
\text { thermodenitrificans } \\
\text { strain KCTC3902 }\end{array}$ & $13,618.31$ & $\begin{array}{l}\text { Geobacillus thermodenitrificans } \\
\text { strain KCTC3902 }\end{array}$ & 100.00 \\
\hline & & & & & & $\begin{array}{l}\text { Geobacillus sp. } \\
\text { PA-3 GEPA3 }\end{array}$ & 100.00 \\
\hline & & & & & & $\begin{array}{l}\text { Geobacillus thermodenitrificans } \\
\text { NG80-2 }\end{array}$ & 100.00 \\
\hline & $\begin{array}{l}\text { B_mud_LB_ } \\
\mathrm{pH} 8\end{array}$ & $\begin{array}{l}\text { Geobacillus vulcani } \\
\text { PSS1 }\end{array}$ & 99.40 & $\begin{array}{l}\text { Geobacillus vulcani } \\
\text { PSS1 }\end{array}$ & $12,550.22$ & $\begin{array}{l}\text { Geobacillus vulcani } \\
\text { PSS1 }\end{array}$ & 100.00 \\
\hline & & & & & & $\begin{array}{l}\text { Geobacillus sp. } \\
\text { FW23 }\end{array}$ & 100.00 \\
\hline \multirow[t]{11}{*}{ B } & NFOSA3 & $\begin{array}{l}\text { Geobacillus galactosidasius } \\
\text { strain DSM } 18751\end{array}$ & 98.85 & $\begin{array}{l}\text { Geobacillus galactosidasius } \\
\text { strain DSM } 18751\end{array}$ & 8003.04 & $\begin{array}{l}\text { Geobacillus galactosidasius } \\
\text { strain DSM } 18751\end{array}$ & 100.00 \\
\hline & DSP4a & $\begin{array}{l}\text { Geobacillus lituanicus } \\
\text { strain N-3 }\end{array}$ & 98.55 & $\begin{array}{l}\text { Geobacillus lituanicus } \\
\text { strain N-3 }\end{array}$ & 4299.05 & $\begin{array}{l}\text { Geobacillus stearothermophilus } \\
\text { strain FHS-PHGT51 }\end{array}$ & 100.00 \\
\hline & & & & & & $\begin{array}{l}\text { Geobacillus stearothermophilus } \\
\text { strain DSM } 458\end{array}$ & 100.00 \\
\hline & & & & & & $\begin{array}{l}\text { Geobacillus stearothermophilus } \\
\text { strain GS27 }\end{array}$ & 100.00 \\
\hline & & & & & & $\begin{array}{l}\text { Geobacillus sp. } \\
\text { Sah69 }\end{array}$ & 100.00 \\
\hline & & & & & & $\begin{array}{l}\text { Geobacillus stearothermophilus } \\
\text { ATCC } 12980\end{array}$ & 100.00 \\
\hline & & & & & & $\begin{array}{l}\text { Geobacillus stearothermophilus } \\
\text { ATCC } 7953\end{array}$ & 100.00 \\
\hline & $\begin{array}{l}\text { CHMud_ } \\
\text { LB_pH8 }\end{array}$ & Anoxybacillus sp. 103 & 98.43 & Anoxybacillus sp. 103 & 7800.76 & $\begin{array}{l}\text { Anoxybacillus kamchatkensis } \\
\text { strain G10 }\end{array}$ & 100.00 \\
\hline & $\begin{array}{l}\text { Efil_LB_ } \\
\text { pH6.8_a }\end{array}$ & $\begin{array}{l}\text { Anoxybacillus ayderensis } \\
\text { strain AB04 }\end{array}$ & 98.40 & $\begin{array}{l}\text { Anoxybacillus ayderensis } \\
\text { strain AB04 }\end{array}$ & $10,221.31$ & $\begin{array}{l}\text { Anoxybacillus kamchatkensis } \\
\text { strain G10 }\end{array}$ & 99.66 \\
\hline & $\begin{array}{l}\text { MMMud_3_- } \\
\text { LB_pH8 }\end{array}$ & $\begin{array}{l}\text { Geobacillus subterraneus } \\
\text { PSS2 }\end{array}$ & 98.14 & $\begin{array}{l}\text { Geobacillus subterraneus } \\
\text { PSS2 }\end{array}$ & 3684.00 & $\begin{array}{l}\text { Geobacillus icigianus } \\
\text { strain G1w1 }\end{array}$ & 99.76 \\
\hline & & & & & & $\begin{array}{l}\text { Geobacillus subterraneus PSS2 } \\
\text { N671 }\end{array}$ & 99.76 \\
\hline $\mathrm{C}$ & $\begin{array}{l}\text { MCWH_LB_ } \\
\mathrm{pH} 8\end{array}$ & $\begin{array}{l}\text { Brevibacillus thermoruber } \\
\text { PM1 N690 }\end{array}$ & 94.13 & $\begin{array}{l}\text { Brevibacillus thermoruber } \\
423\end{array}$ & 3758.04 & $\begin{array}{l}\text { Brevibacillus thermoruber } \\
\text { PM1 N690 }\end{array}$ & 99.35 \\
\hline
\end{tabular}

In each case the same species was identified as the closest match, and in seven of eight cases, the same assembly was identified. For the isolate MCWH_LB_pH8 the two methods identified different assemblies of Brevibacillus thermoruber. In all eight cases the progressiveMauve [35, 36] whole-genome identifications and the reduced-representation sequence results identified a single closest matching candidate assembly. The identification results from the $16 \mathrm{~S}$ rRNA gene sequences sometimes identified multiple closest matching assemblies of equal bitscore and percentage identity. Out of the eight isolates, three did not produce the same species identification results between progressiveMauve [35, 36] and 16S rRNA gene alignment. Additionally, four out of the eight isolates did not identify a single best candidate assembly, based on bitscore and percentage identity. The genome sequences showed that the best $16 \mathrm{~S}$ rRNA gene alignment did not always match the results from wholegenome sequencing; however, misalignments may indicate an intensive horizontal gene transfer or genome rearrangements rather than phylogenetic diversity.

The sequence fragments produced by reduced representation sequencing represented coverage of approximately $2.3 \%$ of the genome, derived from up to 2500 individual fragments, depending on the combination of restriction enzymes used. This number of fragments can be sequenced to a read depth of 40x using 100,000 reads per assay, and the sequence barcoding system allows for multiplexing of up to 2300 assays. The volume of sequencing required to achieve full coverage of the 
reduce-representation fragments is very low in comparison to the sequencing output of current next-generation sequencers. A single lane of a HiSeq 2500 v4 flow-cell could be used to sequence assays for 2300 isolates with a read depth of $>40 x$ across the available fragments. Alternatively, for lower throughput processing, 250 assays could be processed in a MiSeq v3 flow-cell at a 40x read depth. The assay cost per sample, including library construction and sequencing would be expected to be 7 dollars (USD) [37].

\section{Comparison of identification methods}

DNA sequence-based bacterial identification has relied almost exclusively on partial or complete $16 \mathrm{~S}$ ribosomal RNA gene sequencing [38-42]. In spite of the ubiquitous use of $16 \mathrm{~S}$ sequence data, the limitations of this approach are well established [43]. One of the first problems identified with this technique was the difficulty of primer design, necessitating attempts at creation of 'universal' primers, ideally capable of amplifying a portion of the $16 \mathrm{~S}$ rRNA gene from any bacterial isolate [44]. In practice, multiple primer pairs may sometimes need to be trialled to obtain successful amplification from any given isolate. Another issue relates to the limited resolution of the identification information provided by $16 \mathrm{~S}$ rRNA gene sequencing [43]. The original rationale for the choice of the $16 \mathrm{~S}$ rRNA gene for use in bacterial identification is based on the need for a balance between sequence conservation versus sequence diversity. Sequence similarity between taxa must be sufficient for priming and PCR amplification, but sequence variability must be sufficient to provide resolution between taxa for identification purposes. In practice the resolution provided by $16 \mathrm{~S}$ rRNA sequence data can be insufficient for species delimitation as they may be identical between species [45]. Effectively, the sequence similarity of the $16 \mathrm{~S}$ locus may not be a surrogate for the similarity of the genome as a whole [46]. Obtaining a whole-genome sequence is clearly the best option to identify bacterial isolates and determine their nearest relatives; however, the costs involved mean that it is generally not practical to do this for all of bacterial isolates in a study.

An alternative identification method of sequencing complexity-reduced genomic representations could provide a potential replacement, avoiding some of the limitations. This study has shown that reducedrepresentation sequencing can provide fine scale identification information, most importantly, with complete agreement to whole-genome sequence information in terms of identification for the bacterial isolates tested in this study. Reduced-representation sequences can be produced for any organism, without need for prior sequence information, and with no prior knowledge of taxonomic affinities required.

\section{Conclusions}

This study clearly demonstrates the accuracy of the identifications based on reduced-representation sequencing. The eight isolates were selected for whole-genome sequencing to test the accuracy of the preliminary identifications. In each case the closest matching genomes identified by reduced-representation sequencing agreed completely with the identifications provided by wholegenome sequencing. The identifications provided by $16 \mathrm{~S}$ rRNA gene sequence alignment, were in agreement for some of the isolates but differed from the wholegenome-based results for others. The 16S rRNA gene results also identified multiple accessions or even multiple species with equal distance in $50 \%$ of cases, failing to identify a single best candidate from the sequence database. The method of reduced-representation sequencing has been successfully applied in identification of bacterial isolates in a medical microbiology context [37] and this is the first successful use for identification of thermophilic bacterial isolates.

The genome coverage obtained in this study ranged between 1.10 to $4.54 \%$. This coverage is derived from short fragments obtained from across the entire genome, rather than longer consecutive regions, as shown in the circular graph plotted by Circos [33] from Fig. 1. This potentially allows detection of horizontal transfer between taxa; however, in practice many horizontal gene transfer events may be undetectable in the results produced from this method. Further work will need to be done to clarify the limits of horizontal gene transfer detection and the implications for bacterial identifications.

Reduced-representation sequencing is equally well suited for use with small numbers of isolates or with large batch processing of thousands of assays. The growing database of available bacterial genome sequences provides an excellent resource for alignment of reducedrepresentation sequences for identification purposes, and as the available genomes continue to grow, the technique will become more effective with time.

\section{Materials and methods Sampling Hot water systems}

Water samples were collected from nine domestic hot water systems with at least five years of operation in the region of the ACT, Australia. A volume of $1.5 \mathrm{~L}$ was collected into sterile containers, temperature was recorded, and bottles were transported without refrigeration. The water samples were filtered with sterile membranes of $0.20 \mu \mathrm{m}$ pore size (Nalgene ${ }^{\mathrm{Tw}}$ Rapid-Flow $^{\mathrm{Tm}}$, PES Membrane Cat. No. 6.302336, type 565, ThermoFisher Scientific, Australia), connected to a vacuum pump, to capture any bacteria present. 


\section{Commercial composts}

Samples were collected from seven commercial composts. The composts were made from various combinations of animal manure and plant matter. Samples taken from bagged compost were placed in $50 \mathrm{ml}$ sterile falcon tubes and transferred to the laboratory for inoculation.

\section{Artesian bores in the great Artesian Basin, South Australia}

A total of 10 water samples were collected from the tap at the bore head into sterile bottles from selected bore locations on the Birdsville Track, South Australia (Fig. 2 and Supplementary Material 1). A volume of $1.5 \mathrm{~L}$ was taken, temperature was recorded, and water samples were filtered to collect any bacteria present, using the same method described above for hot water systems. In addition, sediment samples were collected from each of the bore drains at various distances from the bore head along the temperature gradient, especially where colonies of microorganisms were visible. These were collected into $50 \mathrm{ml}$ sterile falcon tubes.

\section{Medium composition and cultivation}

Four types of solid culture media were prepared using two different nutrient recipes and a series of $\mathrm{pH}$ values. The first nutrient recipe contained LB medium, and was prepared by dissolving $20 \mathrm{~g} \mathrm{Lb}$ Broth (Sigma-Aldrich L3022) and $5 \mathrm{~g}$ Gelzan $^{\text {tx }}$ CM Gelrite ${ }^{\circ}$ solidifying agent (G1910 Sigma-Aldrich) in $900 \mathrm{ml}$ of miliQ water, then filling up with miliQ water to $1000 \mathrm{ml}$. Culture media were adjusted for final $\mathrm{pH}$ values of 5.0, 6.8 and 8.0. The second nutrient recipe combined $1.0 \mathrm{~g} \mathrm{~L}^{-1}$ yeast extract, $1.0 \mathrm{~g} \mathrm{~L}^{-1}$ tryptone, with a basal medium containing $1.3 \mathrm{~g}$ $\left(\mathrm{NH}_{4}\right)_{2} \mathrm{SO}_{4}, 0.47 \mathrm{~g} \mathrm{~K}_{2} \mathrm{HPO}_{4} \cdot 3 \mathrm{H}_{2} \mathrm{O}, 0.25 \mathrm{~g} \mathrm{MgSO}_{4} \cdot 7 \mathrm{H}_{2} \mathrm{O}$, $0.07 \mathrm{~g} \mathrm{CaCl}_{2}$ and $1 \mathrm{ml}$ of trace element solution [47]. This was prepared by dissolving all components with $5 \mathrm{~g}$ Gelzan $^{\text {Th }}$ CM Gelrite ${ }^{\circ}$ solidifying agent in $900 \mathrm{ml}$ of miliQ water, then filling up with miliQ water to $1000 \mathrm{ml}$ and adjusting to $\mathrm{pH}$ 6.0. All media were autoclaved at $121^{\circ} \mathrm{C}$ for $20 \mathrm{~min}$.

The four variations of culture media and $\mathrm{pH}$ were inoculated with each of the compost, filter strips, and

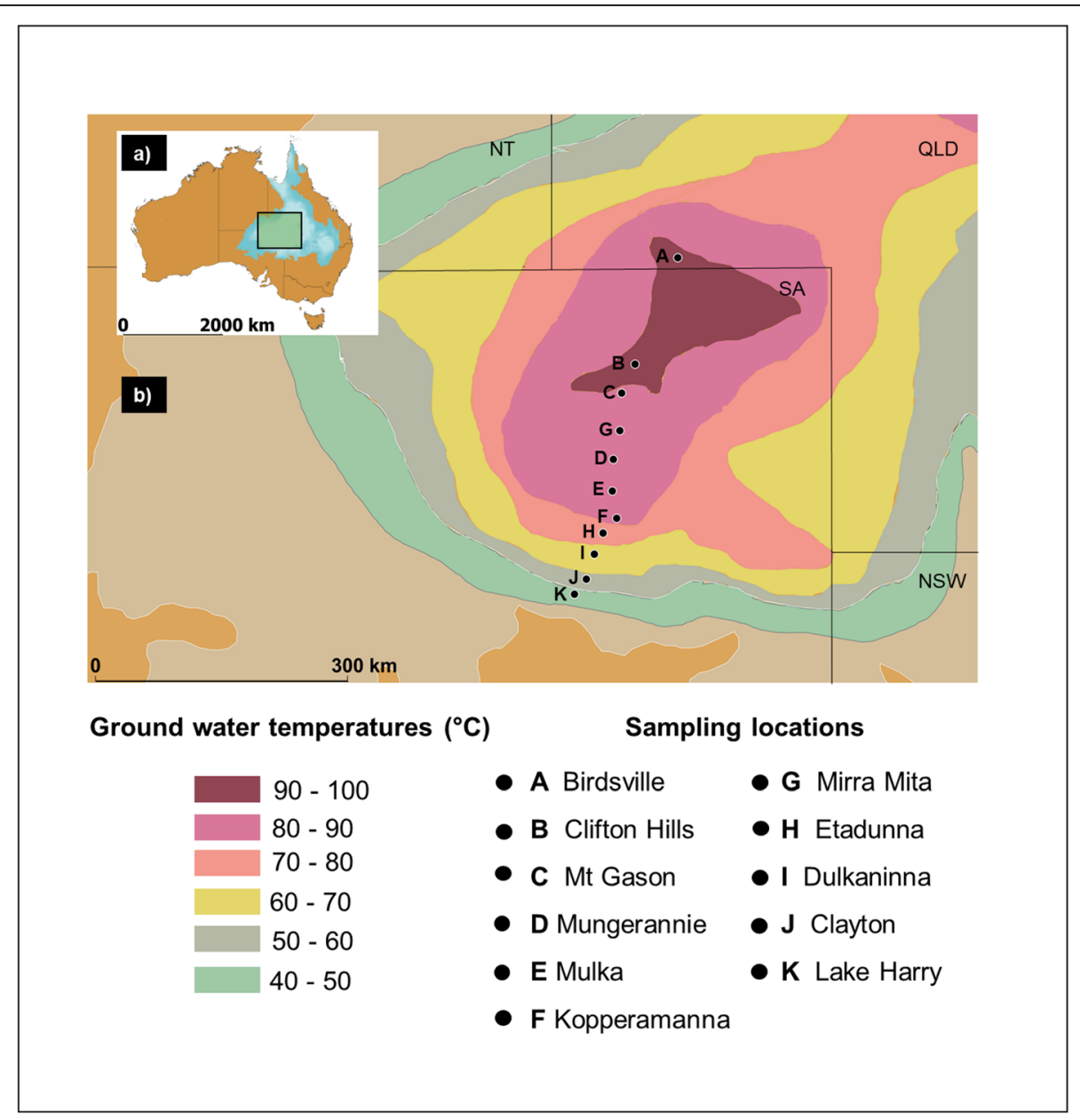

Fig. 2 Sampling locations of 11 water-bores of The Great Artesian Basin showing groundwater temperatures derived from Habermehl and Pestov (2002) [14]. Inset map shows larger position of larger map within Australia 
sediment samples, and all cultures were incubated at $62.5^{\circ} \mathrm{C}$. Filter membranes were removed from the filter, cut into strips with a sterile scalpel blade, and placed inverted on the surface of the solid media. Similarly, approximately $1 \mathrm{~g}$ of compost/sediment was scattered on the surface of the media. Humidity conditions for the culture plates were controlled by adding sterile wet gauzes inside of a sealed plastic box that contained all Petri dishes. Bacterial growth was observed within a range of time from 48 to $72 \mathrm{~h}$. Individual colonies identified were isolated by at least three passages of subculturing from single cell derived colonies.

\section{Library preparation and sequencing}

DNA extractions were performed for all bacterial isolates using the chloroform-isoamyl alcohol method [37]. The library preparation was done following the DArTseq ${ }^{\mathrm{Tm}}$ methods, in which the DNA was digested with pairs of restriction enzymes, in this case, PstI with HpaII and PstI with $M s e$ I respectively, and PCR adapters were ligated to the fragments. Two adapters were used, one corresponding to each restriction enzyme. The adapter design included Illumina flow-cell specific sequences required for bridge PCR in cluster generation, as well as a barcode region to enable sample multiplexing. The adapters were designed such that only fragments with differing restriction sites at each end were capable of cluster generation. Digestion/ligation was followed by PCR amplification according to Georges et al. (2018). Final PCR products were stored at $4{ }^{\circ} \mathrm{C}$. A post PCR quality control was performed by agarose gel electrophoresis. An equal volume of PCR product from each sample was pooled and then purified with a QIAGEN QIAquick PCR Purification Kit Cat. ID: 28106 (QUIAGEN, Chatstone, Victoria, Australia). For each sample, libraries made with both complexityreduction methods were pooled together for sequencing. Clustering was done according to Illumina HiSeq SR Cluster Kit V4 recipe v9.0 and HiSeq SR Flow Cell v4 (Illumina Inc., San Diego CA, US). For sequencing, the Flow Cell was loaded according to the Illumina protocols on a HiSeq 2500 sequencer, using HiSeq SBS kit v4 for a total of 77 cycles [30].

A control was also sequenced, using the genomic DNA of Eschericha coli O157 (EDL 933) IRMM449 Sigma-Aldrich (Castle Hill, NSW, Australia) certified reference standard, GenBank [47] accession number AE005174.2, genome size of 5,639,399 bp [31]. This control was processed for library construction, sequencing and analysis using methods identical to those used for all other bacterial isolates.

\section{Data analysis}

Data from the sequencer in the form of FastQ files was processed according to the methods described in
Talamantes-Becerra et al. (2019). Briefly, sequences were filtered by PHRED quality score, barcode sequences were removed and identical sequences were recognised and collapsed into 'fastQcol' files, which contain each unique sequence present in the original FastQ file, along with the respective read counts and the mean quality score at each base [48]. The reverse adapters which were present on sequences derived from fragments shorter than $69 \mathrm{bp}$ were identified and trimmed, resulting in sequences from $30 \mathrm{bp}$ to $69 \mathrm{bp}$.

Data for each of the complexity reduction methods was processed with the analytical pipeline Currito3.1 DNA Fragment Analysis Software [32], which we developed specifically for analysing reduced-representation sequences from bacteria. The details of this software pipeline are described in Talamantes-Becerra et al. (2019). Briefly, a BLASTn alignment [49] of the trimmed sequence tags from each sample against all complete bacterial genome assemblies from the NCBI RefSeq database is performed, to identify the best candidate bacterial genomes for each sample. The BLASTn parameters were used were: word size 12 , bitscore 50 , evalue 0.000001 , percentage identity 80 , percentage query cover $80 \%$. Candidate matching genome assemblies are selected according to the number of sequence tags obtaining a best or equal best BLASTn hit to each reference, as measured by bit score. After identifying candidate genomes, the trimmed sequences from each sample are aligned by BLASTn against the top three closest identified genomes individually. The Currito3.1 [32] pipeline uses the NSD calculation, shown in the following equation to determine the best matching candidate genome for each sample, based on the BLASTn alignments described above.

$$
N S D=-\frac{3}{4} \ln \left[1-\frac{4}{3}\left(\frac{S}{I+S}\right)\right]\left[1-\frac{G}{T}\right]+\frac{G}{T}
$$

NSD is a DNA sequence distance measurement considering identities (I), substitutions (S) and gap openings (G) across all aligned sequences to produce a global distance value [50]. Closer relatedness to a reference genome is associated with lower NSD values.

\section{Confirmation of strain identification results by whole- genome sequencing}

The genomic DNA of selected isolates was purified using the Agencourt AMPure XP (Beckman Coulter Inc., Brea, CA, US) genomic DNA purification beads protocol. The volume of Agencourt AMPure XP beads used for purification was $0.4 \mathrm{x}$ genomic DNA volume. Briefly, genomic DNA clean-up was done as follows: selected volumes of AMPure XP beads and genomic DNA was mixed by pipette, then incubated for $5 \mathrm{~min}$ at room 
temperature. Sample tubes were placed onto the magnetic plate for $2 \mathrm{~min}$, and supernatant was discarded carefully leaving $5 \mu \mathrm{L}$. Then $200 \mu \mathrm{L}$ of freshly prepared $70 \%$ ethanol was added to the beads, incubating for $30 \mathrm{~s}$ before removal of the supernatant over the magnetic plate. This wash step was repeated. After the second wash, ethanol was completely removed and beads were allowed to dry. Samples were removed from the magnetic plate, eluted in $35 \mu \mathrm{L}$ of EB buffer $(10 \mathrm{mM}$ Tris-Cl, $\mathrm{pH} 8.5$ ), and mixed by pipette 10 times. Sample tubes were located again onto the magnetic plate for $1 \mathrm{~min}$ and elution buffer was transferred into a new tube.

The whole-genome sequencing service was provided by MicrobesNG, IMI - School of Biosciences, University of Birmingham, United Kingdom. Libraries for wholegenome sequencing were prepared with the Nextera XT Library Prep Kit (Illumina, San Diego, USA), then quantified with the Microlab STAR automated liquid handling system. All libraries were quantified and pooled for sequencing with the Kapa Biosystems Library Quantification Kit for Illumina on a Roche light cycler 96 qPCR machine and were sequenced on the Illumina HiSeq2500 using a $250 \mathrm{bp}$ paired end protocol. Sequencing depth for all bacterial isolates was 30X. To process the sequence data, Trimmomatic 0.30 [51] was used for trimming reads, SPAdes version 3.7 [52] was used for de novo assembly, and Prokka 1.11 [53] was used for annotation of contigs.

The resulting draft genome assemblies for the 8 isolates were assessed using the software tool Kraken [54], which utilises DNA sequence K-mer alignments to determine taxonomic affinities. The Kraken results placed all isolates within the same genera reported by the Currito3.1 [32] analytical pipeline.

The draft genome assemblies were then aligned against all available Anoxybacillus, Geobacillus and Brevibacillus assemblies totalling 31, 72 and 63 genome assemblies respectively, from GenBank [55] database up to December 2018, to identify the closest matching genome assembly for each isolate. For alignment of the wholegenome assemblies, the software progressiveMauve [35, 36] was used.

For each of the eight draft genomes, the pairwise alignment which resulted in the highest mean similarity profile value was considered the closest matching genome. The identifications obtained in this way were used to test the accuracy of the identifications obtained using the method under current investigation, based on reduced-representation sequencing. In addition to the genome alignments, the complete $16 \mathrm{~S}$ rRNA gene CDS sequence was also used to identify the closest genome assembly from amongst the same set of available RefSeq [55] assemblies. For each of the eight isolates, the $16 \mathrm{~S}$ rRNA gene sequence was obtained from the draft genome assembly using the annotation produced by Prokka 1.11 [53]. The complete 16S rRNA gene sequences were aligned by BLASTn against each of the RefSeq Anoxybacillus, Geobacillus and Brevibacillus genome assemblies in order to find the best or equal best matches for the 16S rRNA gene, along with percentage identity values.

\section{Supplementary information}

Supplementary information accompanies this paper at https://doi.org/10. 1186/s12866-020-01800-z.

\section{Additional file 1.}

\section{Abbreviations}

16S rRNA: 165 ribosomal RNA; ACT: Australian Capital Territory; ANI: Average Nucleotide Identity; BLASTn: Basic Local Alignment Search Tool (Nucleotides); E. coli: Escherichia coli; LCBs: Locally Colinear Blocks; NCBI: National Center for Biotechnology Information; NSD: Nucleotide Sequence Distance;

PCR: Polymerase Chain Reaction

\section{Acknowledgements}

We would like to thank Dr. M.A.(Rien) Habermehl for sharing his expertise and advice during the selection of sampling sites and indicating the best season for collecting samples in the Great Artesian Basin. We would like to thank D. Shrestha for collecting and sending back to us, mud and water samples from Birdsville and Stoney crossing artesian water bores. We would like to thank the station managers from the Birdsville Track for allowing us to collect samples from their artesian water bores. We thank Dr. Michelle Gahan and Prof. Dennis McNevin for their suggestions on project development and methods and for co-supervising the PhD project from which this work arises.

\section{Authors' contributions}

BTB and JC created bioinformatic pipeline for data analysis. AG provided primary supervision of the PhD project from which this paper arose, critically revised the manuscript and contributed expertise in statistical analysis. AK co-supervised the PhD project from which this work arises, revised the methods and provided statistical advice. BTB conceived the project, undertook laboratory work and led the writing of the manuscript. All the authors have read, contributed and approved the manuscript.

\section{Funding}

BTB was awarded a PhD scholarship from Consejo Nacional de Ciencia y Tecnología 'CONACYT - Becas CONACYT al extranjero 2015' programme. The funding body had no role in study design, sample collection, analysis, interpretation of data, writing the manuscript, and decision to publish.

\section{Availability of data and materials}

Scripts for Currito3.1 DNA Fragment Analysis Software are available on GitHub. https://github.com/BTalamantesBecerra/Currito3.1 https://doi.org/10. 5281/zenodo.3748447

The datasets generated and/or analysed during the current study are available on Figshare Repository. DOl: https://doi.org/10.6084/m9.figshare. 11930892

This Whole-Genome Shotgun project has been deposited at DDBJ/ENA/GenBank under the accessions SDLA00000000, SDLB00000000, SDLC00000000, SDLD00000000, SDLE00000000, SDLF00000000, SDLG00000000, SDLH00000000. The raw FASTQ reads have been deposited in the NCBI SRA database under the accession numbers SRR8490233, SRR8490232, SRR8490231, SRR8490230, SRR8490229, SRR8490228, SRR8490227, SRR8490226.

Ethics approval and consent to participate "Not applicable". 


\section{Competing interests}

AK is the director of Diversity Arrays Technology (DArT), JC is a full-time employee of DArT and BTB is enrolled in a PhD which involves the use of DArT Technology. AG declares no competing interests.

\section{Author details}

${ }^{1}$ Institute of Applied Ecology, University of Canberra, Canberra, ACT 2601, Australia. ${ }^{2}$ Diversity Arrays Technology Pty Ltd, Canberra, ACT 2617, Australia.

Received: 5 June 2019 Accepted: 23 April 2020

Published online: 13 May 2020

\section{References}

1. Greaves RB, Warwicker J. Stability and solubility of proteins from extremophiles. Biochem Biophys Res Commun. 2009;380:581-5. https://doi. org/10.1016/j.bbrc.2009.01.145.

2. Elleuche S, Schäfers C, Blank S, Schröder C, Antranikian G. Exploration of extremophiles for high temperature biotechnological processes. Curr Opin Microbiol. 2015;25:113-9. https://doi.org/10.1016/j.mib.2015.05.011.

3. Urbieta MS, Donati ER, Chan KG, Shahar S, Sin LL, Goh KM. Thermophiles in the genomic era: biodiversity, science, and applications. Biotechnol Adv. 2015;33:633-47. https://doi.org/10.1016/j.biotechadv.2015.04.007.

4. Mehta $R$, Singhal $P$, Singh $H$, Damle D, Sharma AK. Insight into thermophiles and their wide-spectrum applications. 3. Biotech. 2016;6:1-9. https://doi.org/ 10.1007/s13205-016-0368-z.

5. Krüger A, Schäfers C, Schröder C, Antranikian G. Towards a sustainable biobased industry - highlighting the impact of extremophiles. New Biotechnol. 2018;40:144-53. https://doi.org/10.1016/j.nbt.2017.05.002.

6. Memarpoor-Yazdi M, Karbalaei-Heidari HR, Doroodmand MM. Enantioselective hydrolysis of ibuprofen ethyl ester by a thermophilic immobilized lipase, ELT, from Rhodothermus marinus. Biochem Eng J. 2018; 130:55-65. https://doi.org/10.1016/j.bej.2017.11.016.

7. Memarpoor-Yazdi M, Karbalaei-Heidari HR, Khajeh K. Production of the renewable extremophile lipase: valuable biocatalyst with potential usage in food industry. Food Bioprod Process. 2017;102:153-66. https://doi.org/10. 1016/j.fbp.2016.12.015

8. Narwal SK, Saun NK, Dogra P, Gupta R. Green synthesis of isoamyl acetate via silica immobilized novel thermophilic lipase from Bacillus aerius. Russ J Bioorganic Chem. 2016;42:69-73. https://doi.org/10.1134/ S1068162016010118.

9. Raza FA, Sabri AN, Rehman A, Hasnain S. Characterization of thermophilic alkaline lipase produced by Staphylococcus aureus suitable for leather and detergent industries. Iran J Sci Technol Trans A Sci. 2017;41:287-94. https:// doi.org/10.1007/s40995-017-0265-2

10. Mehta D, Satyanarayana T. In: Satyanarayana T, Littlechild J, Kawarabayasi Y, editors. Diversity of hot environments and thermophilic microbes thermophilic microbes in environmental and industrial biotechnology: biotechnology of thermophiles. Dordrecht: Springer Netherlands; 2013. p. 3-60. https://doi.org/10.1007/978-94-007-5899-5_1.

11. Wynter C, Patel BKC, Bain P, De Jersey J, Hamilton S, Inkerman PA. A novel thermostable dextranase from a Thermoanaerobacter species cultured from the geothermal waters of the great Artesian Basin of Australia. FEMS Microbiol Lett. 1996;140:271-6. https:/doi.org/10.1016/0378-1097(96)00193-0.

12. Byers HK, Stackebrandt E, Hayward C, Blackall LL. Molecular investigation of a microbial mat associated with the great Artesian Basin. FEMS Microbiol Ecol. 1998;25:391-403. https://doi.org/10.1016/S0168-6496(98)00013-0.

13. Kimura H, Sugihara M, Yamamoto H, Patel BKC, Kato K, Hanada S. Microbial community in a geothermal aquifer associated with the subsurface of the great Artesian Basin. Australia Extremophiles. 2005;9:407-14. https://doi.org/ 10.1007/s00792-005-0454-3.

14. Habermehl MA, Pestov I. Geothermal resources of the great Artesian Basin, Australia. Geo-Heat Center Quarterly Bulletin. 2002;23:20-6 https://www.oit. edu/orec/geo-heat-center/quarterly-bulletin. Accessed 9 May 2019.

15. Habermehl MA. Hydrogeology and environmental geology of the Great Artesian Basin, Australia. In: Gostin VA, editor. Geological Society of Australia Special Publication; 1999. p. 127-43

16. Ogg CD, Spanevello MD, Patel BKC. In: Satyanarayana T, Littlechild J, Kawarabayasi $Y$, editors. Exploring the ecology of thermophiles from Australia's great Artesian Basin during the genomic era - thermophilic microbes in environmental and industrial biotechnology: biotechnology of thermophiles. Dordrecht: Springer Netherlands; 2013. p. 61-97. https://doi. org/10.1007/978-94-007-5899-5_2.

17. Strom PF. Identification of thermophilic bacteria in solid-waste composting. Appl Environ Microbiol. 1985:50:906-13.

18. Poli A, Laezza G, Gul-Guven R, Orlando P, Nicolaus B. Geobacillus galactosidasius sp. nov., a new thermophilic galactosidase-producing bacterium isolated from compost. Syst Appl Microbiol. 2011;34:419-23. https://doi.org/10.1016/j.syapm.2011.03.009.

19. Finore I, Gioiello A, Leone L, Orlando P, Romano I, Nicolaus B, et al. Aeribacillus composti sp. nov., a thermophilic bacillus isolated from olive mill pomace compost. Int J Syst Evol Microbiol. 2017;67:4830-5. https://doi. org/10.1099/ijsem.0.002391.

20. Lemos LN, Pereira RV, Quaggio RB, Martins LF, Moura LMS, da Silva AR, et al. Genome-centric analysis of a thermophilic and cellulolytic bacterial consortium derived from composting. Front Microbiol. 2017;8:1-16. https:// doi.org/10.3389/fmicb.2017.00644.

21. Brock T, Boylen K. Presence of thermophilic bacteria in laundry and domestic hot-water heaters. Appl Microbiol. 1973;25:72-6.

22. Bagh LK, Albrechtsen HJ, Arvin E, Ovesen K. Distribution of bacteria in a domestic hot water system in a Danish apartment building. Water Res. 2004:38:225-35. https://doi.org/10.1016/j.watres.2003.08.026.

23. Jain C, Rodriguez-R LM, Phillippy AM, Konstantinidis KT, Aluru S. High throughput ANI analysis of 90K prokaryotic genomes reveals clear species boundaries. Nat Commun. 2018;9:5114. https://doi.org/10.1038/s41467-01807641-9.

24. Kilian A, Wenzl P, Huttner E, Carling J, Xia L, Blois H, et al. Diversity arrays technology: a generic genome profiling technology on open platforms. Methods Mol Biol. 2012;888:67-89. https://doi.org/10.1007/978-1-61779-870-2_5.

25. dos Santos JPR, Pires LPM, de Castro Vasconcellos RC, Pereira GS, Von Pinho RG, Balestre M. Genomic selection to resistance to Stenocarpella maydis in maize lines using DArTseq markers. BMC Genet. 2016;17:1-10. https://doi. org/10.1186/s12863-016-0392-3.

26. Garavito A, Montagnon C, Guyot R, Bertrand B. Identification by the DArTseq method of the genetic origin of the Coffea canephora cultivated in Vietnam and Mexico. BMC Plant Biol. 2016;16:1-12. https://doi.org/10.1186/ s12870-016-0933-y.

27. Baloch FS, Alsaleh A, Shahid MQ, Çiftçi V, Sáenz De Miera LE, Aasim M, et al. A whole genome DArTseq and SNP analysis for genetic diversity assessment in durum wheat from central fertile crescent. PLoS One. 2017;12: 1-18. https://doi.org/10.1371/journal.pone.0167821.

28. Egea LA, Mérida-García R, Kilian A, Hernandez P, Dorado G. Assessment of genetic diversity and structure of large garlic (Allium sativum) germplasm bank, by diversity arrays technology "genotyping-by-sequencing" platform (DArTseq). Front Genet. 2017;8:1-9. https://doi.org/10.3389/fgene.2017. 00098.

29. Lambert MR, Skelly DK, Ezaz T. Sex-linked markers in the north American green frog (Rana clamitans) developed using DArTseq provide early insight into sex chromosome evolution. BMC Genomics. 2016;17:1-13. https://doi. org/10.1186/s12864-016-3209-x.

30. Georges A, Gruber B, Pauly GB, White D, Adams M, Young MJ, et al. Genome-wide SNP markers breathe new life into phylogeography and species delimitation for the problematic short-necked turtles (Chelidae: Emydura) of eastern Australia. Mol Ecol. 2018;27:5195-213. https://doi.org/10. 1111/mec.14925.

31. Perna NT, Plunkett G, Burland V, Mau B, Glasner JD, Rose DJ, et al. Genome sequence of enterohaemorrhagic Escherichia coli O157:H7. Nature. 2001;409: 529-33. https://doi.org/10.1038/35054089.

32. Talamantes-Becerra B, Carling J. Currito3.1 DNA Fragment Analysis Software; 2020. https://doi.org/10.5281/zenodo.3748447.

33. Krzywinski MI, Schein JE, Birol I, Connors J, Gascoyne R, Horsman D, et al. Circos: an information aesthetic for comparative genomics. Genome Res. 2009. https://doi.org/10.1101/gr.092759.109.

34. Fujio $Y$, Kume S. Isolation and identification of thermophilic bacteria from sewage sludge compost. J Ferment Bioeng. 1991;72:334-7. https://doi.org/ 10.1016/0922-338X(91)90082-R.

35. Darling AE, Mau B, Perna NT. progressiveMauve: multiple genome alignment with gene gain, loss and rearrangement. PLOS One. 2010;5: e11147. https://doi.org/10.1371/journal.pone.0011147.

36. Darling ACE, Mau B, Blattner FR, Perna NT. Mauve: multiple alignment of conserved genomic sequence with rearrangements. Genome Res. 2004;14: 1394-403. https://doi.org/10.1101/gr.2289704. 
37. Talamantes-Becerra B, Carling J, Kennedy K, Gahan M, Georges A. Identification of bacterial isolates from a public hospital in Australia using complexity-reduced genotyping. J Microbiol Methods. 2019;160:11-9. https://doi.org/10.1016/j.mimet.2019.03.016.

38. Tomova I, Stoilova-Disheva M, Lyutskanova D, Pascual J, Petrov P, Kambourova M. Phylogenetic analysis of the bacterial community in a geothermal spring, Rupi Basin, Bulgaria. World J Microbiol Biotechnol. 2010; 26:2019-28. https://doi.org/10.1007/s11274-010-0386-7.

39. Sahoo RK, Subudhi E, Kumar M. Investigation of bacterial diversity of hot springs of Odisha, India. Genomics Data. 2015;6:188-90. https://doi.org/10. 1016/j.gdata.2015.09.018.

40. Sakai HD, Kurosawa N. Exploration and isolation of novel thermophiles in frozen enrichment cultures derived from a terrestrial acidic hot spring. Extremophiles. 2016;20:207-14. https://doi.org/10.1007/s00792-016-0815-0.

41. Najar IN, Sherpa MT, Das S, Das S, Thakur N. Microbial ecology of two hot springs of Sikkim: predominate population and geochemistry. Sci Total Environ. 2018;637-638:730-45. https://doi.org/10.1016/j.scitotenv.2018.05.037.

42. Parikka K, Jacquet S, Colombet J, Guillaume D, Le Romancer M. Abundance and observations of thermophilic microbial and viral communities in submarine and terrestrial hot fluid systems of the French southern and Antarctic lands. Polar Biol. 2018;41:1335-52. https://doi.org/10.1007/s00300018-2288-3.

43. Chatellier S, Mugnier N, Allard F, Bonnaud B, Collin V, van Belkum A, et al. Comparison of two approaches for the classification of 165 rRNA gene sequences. J Med Microbiol. 2014;63:1311-5. https://doi.org/10.1099/jmm.0. 074377-0.

44. Weisburg WG, Barns SM, Pelletier DA, Lane DJ. 165 ribosomal DNA amplification for phylogenetic study. J Bacteriol. 1991;173:697-703.

45. Janda JM, Abbott SL. 16S rRNA gene sequencing for bacterial identification in the diagnostic laboratory: pluses, perils, and pitfalls. J Clin Microbiol. 2007: 45:2761-4. https://doi.org/10.1128/JCM.01228-07.

46. Louca S, Doebeli M, Parfrey LW. Correcting for 165 rRNA gene copy numbers in microbiome surveys remains an unsolved problem. Microbiome. 2018;6:1-12. https://doi.org/10.1186/s40168-018-0420-9.

47. Clark K, Karsch-Mizrachi I, Lipman DJ, Ostell J, Sayers EW. GenBank. Nucleic Acids Res. 2016;44:D67-72. https://doi.org/10.1093/nar/gkv1276.

48. Ren R, Ray R, Li P, Xu J, Zhang M, Liu G, et al. Construction of a high-density DArTseq SNP-based genetic map and identification of genomic regions with segregation distortion in a genetic population derived from a cross between feral and cultivated-type watermelon. Mol Gen Genomics. 2015; 290:1457-70. https://doi.org/10.1007/s00438-015-0997-7.

49. McGinnis S, Madden TL. BLAST: at the core of a powerful and diverse set of sequence analysis tools. Nucleic Acids Res. 2004;32 suppl_2:W20-5. https:// doi.org/10.1093/nar/gkh435.

50. Van de Peer Y, Neefs JM, De Wachter R. Small ribosomal subunit RNA sequences, evolutionary relationships among different life forms, and mitochondrial origins. J Mol Evol. 1990;30:463-76.

51. Bolger AM, Lohse M, Usadel B. Trimmomatic: a flexible trimmer for Illumina sequence data. Bioinformatics. 2014;30:2114-20. https://doi.org/10.1093/ bioinformatics/btu170.

52. Bankevich A, Nurk S, Antipov D, Gurevich AA, Dvorkin M, Kulikov AS, et al. SPAdes: a new genome assembly algorithm and its applications to singlecell sequencing. J Comput Biol. 2012;19:455-77. https://doi.org/10.1089/ $\mathrm{cmb} .2012 .0021$.

53. Seemann T. Prokka: rapid prokaryotic genome annotation. Bioinformatics. 2014;30:2068-9. https://doi.org/10.1093/bioinformatics/btu153.

54. Wood DE, Salzberg SL. Kraken: ultrafast metagenomic sequence classification using exact alignments. Genome Biol. 2014;15:2-12. https://doi. org/10.1186/gb-2014-15-3-r46.

55. O'Leary NA, Wright MW, Brister JR, Ciufo S, Haddad D, McVeigh R, et al. Reference sequence (RefSeq) database at NCBI: current status, taxonomic expansion, and functional annotation. Nucleic Acids Res. 2016;44:D733-45. https://doi.org/10.1093/nar/gkv1189.

\section{Publisher's Note}

Springer Nature remains neutral with regard to jurisdictional claims in published maps and institutional affiliations.

\section{Ready to submit your research? Choose BMC and benefit from}

- fast, convenient online submission

- thorough peer review by experienced researchers in your field

- rapid publication on acceptance

- support for research data, including large and complex data types

- gold Open Access which fosters wider collaboration and increased citations

- maximum visibility for your research: over $100 \mathrm{M}$ website views per year

At BMC, research is always in progress.

Learn more biomedcentral.com/submissions 